\title{
Doppler Waveform Pattern Changes in a Patient with Primary Budd-Chiari Syndrome before and after Angioplasty
}

\author{
Kana Hirooka, Masashi Hirooka, Yoshiyasu Kisaka, Takahide Uehara, \\ Yoichi Hiasa, Kojiro Michitaka and Morikazu Onji
}

\begin{abstract}
A 34-year-old woman was admitted for treatment of esophageal varices. Seven years earlier, she had been diagnosed with Budd-Chiari syndrome, and percutaneous transluminal angioplasty (PTA) for right hepatic vein (RHV) stenosis was done. On admission, Doppler sonography showed a flat waveform in the RHV. RHV re-stenosis was confirmed on X-ray sonography. After PTA, the stenosis improved. Heterogeneous liver enhancement on enhanced computed tomography became homogeneous, her esophageal varices became inconspicuous, and the flat Doppler waveform pattern changed to a triphasic pattern. Doppler sonography was very useful for evaluating the effect of angioplasty and to diagnose re-stenosis.
\end{abstract}

Key words: Budd-Chiari syndrome, Doppler sonography, percutaneous transluminal angioplasty

(DOI: 10.2169/internalmedicine.47.0501)

\section{Introduction}

Budd-Chiari syndrome is an uncommon disease characterized by hepatic venous outflow obstruction. Liver congestion leads to centrilobular fibrosis and finally progresses to cirrhosis (1-4). The syndrome has a variety of causes, including: membrane obstruction, venous thrombosis due to hematological or other diseases, and tumor cell invasion into the hepatic vein or inferior vena cava (IVC) (5-9). In Asia, membranous obstruction of the IVC is the most common cause of Budd-Chiari syndrome $(10,11)$. X-ray venography is useful for showing evidence of hepatic vein thrombosis, membranes, and collateral vessels $(12,13)$. The therapeutic options include surgery, interventional angioplasty, and liver transplantation. It is known that blood flow in the hepatic vein, portal vein, and/or IVC is changed by these treatments. Evaluating the blood flow in these vessels helps make the diagnosis, confirm the efficacy of therapy, and identify disease recurrence. We report a patient with Budd-Chiari syndrome treated by angioplasty, in whom Doppler sonography was useful for assessing the efficacy of treatment by allowing the blood flow in the portal vein to be evaluated.

\section{Case Report}

A 27-year-old woman was found to have abnormal liver function in August 1999, but the etiology was not clarified. Two years later, she was admitted to our hospital for further examination of hepatosplenomegaly, ascites, and esophageal varices on computed tomography (CT) and endoscopic examination. Laparoscopic liver biopsy showed congestive liver cirrhosis. X-ray venography revealed stenosis of the right hepatic vein (RHV) and inferior vena cava (IVC) by a membranous structure. The other hepatic veins were completely obstructed. A shunt connected the RHV to the middle and left hepatic veins. She was diagnosed as having Budd-Chiari syndrome. Color Doppler sonography showed a flat waveform in the RHV (Fig. 1a). The percutaneous transluminal angioplasty (PTA) was used to treat the obstruction caused by the membrane in the RHV in this patient; her liver congestion improved (Fig. 2). At one week after PTA, the flat appearance of the waveform changed to a decreased amplitude pattern (Fig. 1b). However, in September 2006, the esophageal varices worsened, and she was again admitted (Fig. 3a). CT showed stenosis of the RHV and heteroge- 


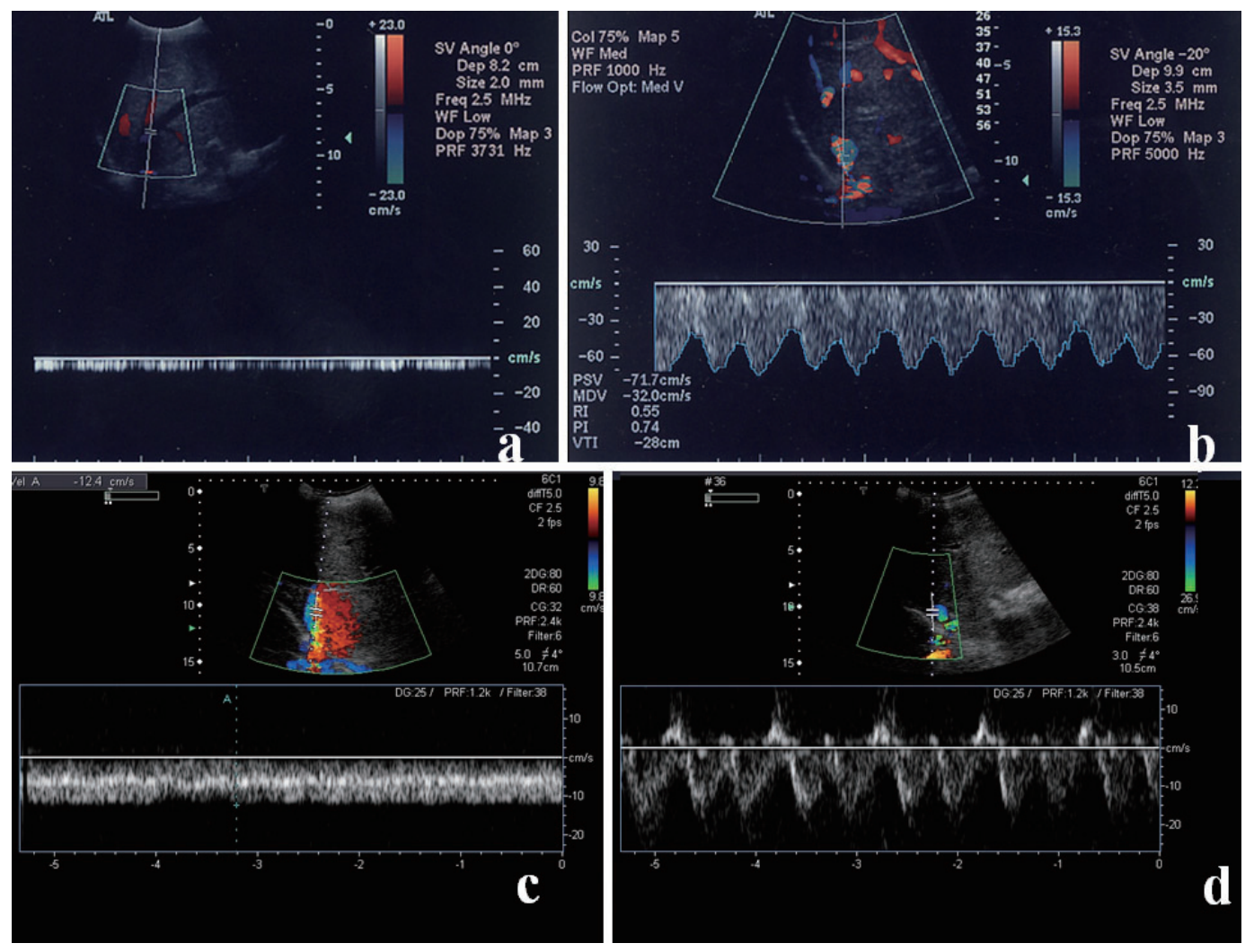

Figure 1. Doppler sonography waveform of the right hepatic vein. During the first admission, a flat pattern was seen (a). A week after the first angioplasty, the RHV waveform showed a decreased amplitude pattern (b). At the time of the second admission, the waveform was similar to that seen on the first admission (c). Just after the second angioplasty, the waveform showed a triphasic pattern (d).
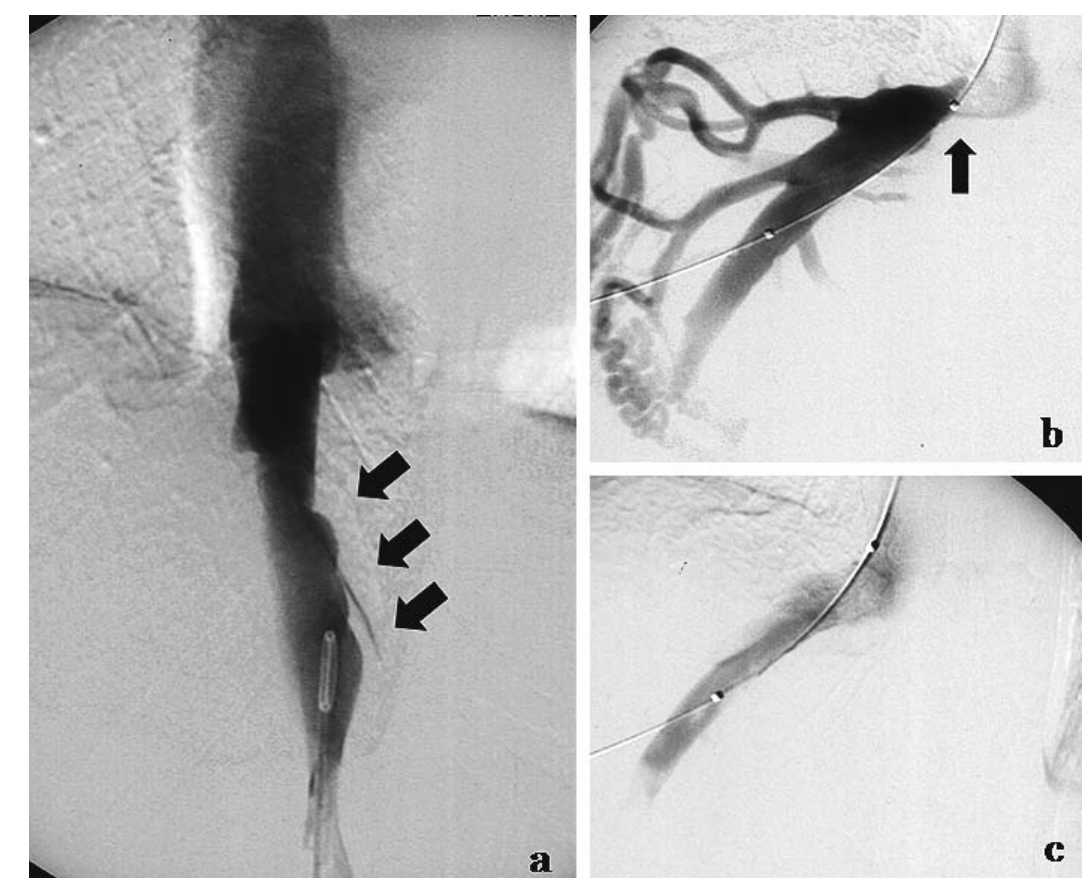

Figure 2. X-ray venography on the first admission showed stenosis of IVC (a) and RHV (b). Just after angioplasty, the stenosis of RHV was improved (c). Arrows indicate the site of stenosis. 


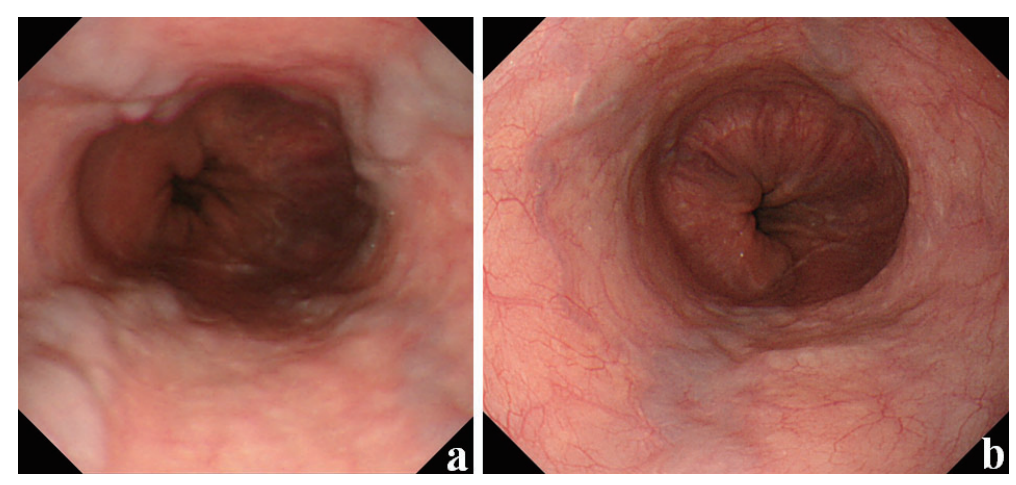

Figure 3. Changes in the esophageal varices seen on endoscopic examination before and after angioplasty. Before treatment, varices showed a beaded appearance (a); at one month after treatment, the varices were straight $(b)$.
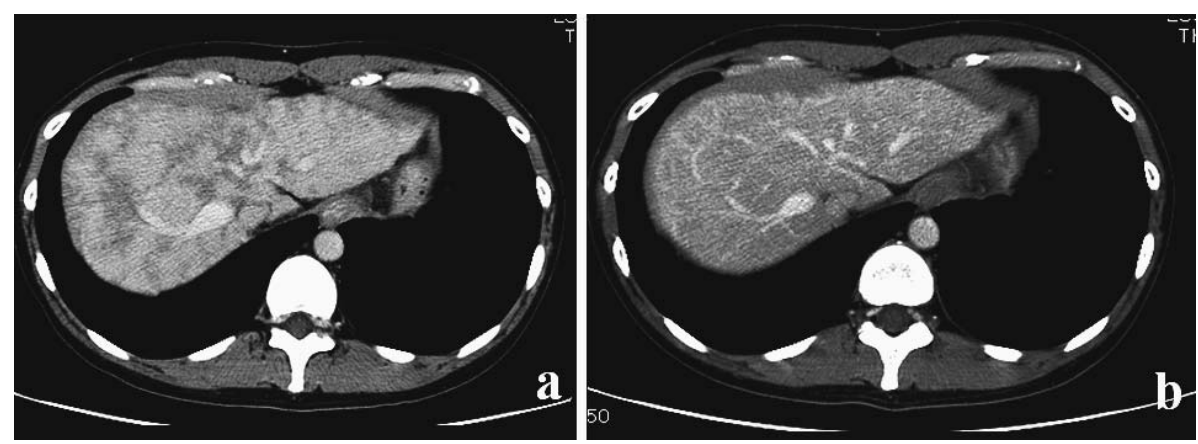

Figure 4. Enhanced computed tomography in the venous phase before and after angioplasty. The liver showed a heterogeneous appearance before angioplasty (a); at one week after treatment, liver congestion was seen to have improved (b).

neous enhancement of the liver (Fig. 4a). Color Doppler sonography showed a rambled pattern in the RHV, and the waveform had a flat appearance rather than the normal triphasic appearance (Fig. 1c). A second angioplasty was done to expand the RHV stenosis (Fig. 5). The hepatic veinIVC wedge pressure in the right hepatic vein decreased from $31.5 \mathrm{mmH}_{2} \mathrm{O}$ before angioplasty to $10.5 \mathrm{mmH}_{2} \mathrm{O}$ after angioplasty. After angioplasty, Doppler sonography showed a normal triphasic waveform in the RHV (Fig. 1d); in the venous phase of the enhanced $\mathrm{CT}$, the irregularity of liver enhancement improved (Fig. 4b), and the esophageal varices also improved (Fig. 3b).

\section{Discussion}

Budd-Chiari syndrome can be either primary or secondary. The primary type is caused by an intrinsic luminal web or thrombosis, while the secondary type is caused by extraluminal compression or tumoral invasion (5-7). Another classification has been proposed based on the location of the venous occlusion. In this classification, membranous obstruction in the IVC is classified as Type I; incomplete obstruction is Ia, and complete obstruction is Ib. Complete obstruction of half of the length of IVC is classified as Type II, complete obstruction of the entire length of the IVC is classified as Type III, and complete obstruction of only the hepatic vein is classified as Type IV. Type Ia is the most frequent, accounting for $34.4 \%$ of cases. Based on these classifications, the present case could be classified as a primary Type Ia. The right hepatic vein had a membranous stenosis, and the other hepatic veins were occluded. This patient did not have a coagulation disorder or antiphospholipid syndrome. The natural history of unrelieved hepatic venous outflow obstruction is progression to liver failure with fibrotic changes. In patients treated supportively, the 1-year mortality of Budd-Chiari syndrome is $25-60 \%$ (14). Therefore, patients with this syndrome require early diagnosis and treatment to release the outflow obstruction.

A few reports have stated that the Doppler waveform of hepatic veins is correlated with the degree of hepatic disease. Bolondi et al noted a relationship between the severity of liver cirrhosis and the disappearance of the phasic waveform (15). They thought that changes in the Doppler pattern were caused by changes in the liver parenchyma that occur with liver cirrhosis. In fact, Ohta et al reported that there was a significant difference in the Child-Pugh score between patients who had a triphasic pattern and those who had a flat pattern with fluttering (16). Thus, a completely flat waveform or no waveform pattern was found only in cases that had hepatic vein stenosis and occlusion.

Loss of the phasic waveform of the portal vein on Doppler sonography is an important finding for making the di- 

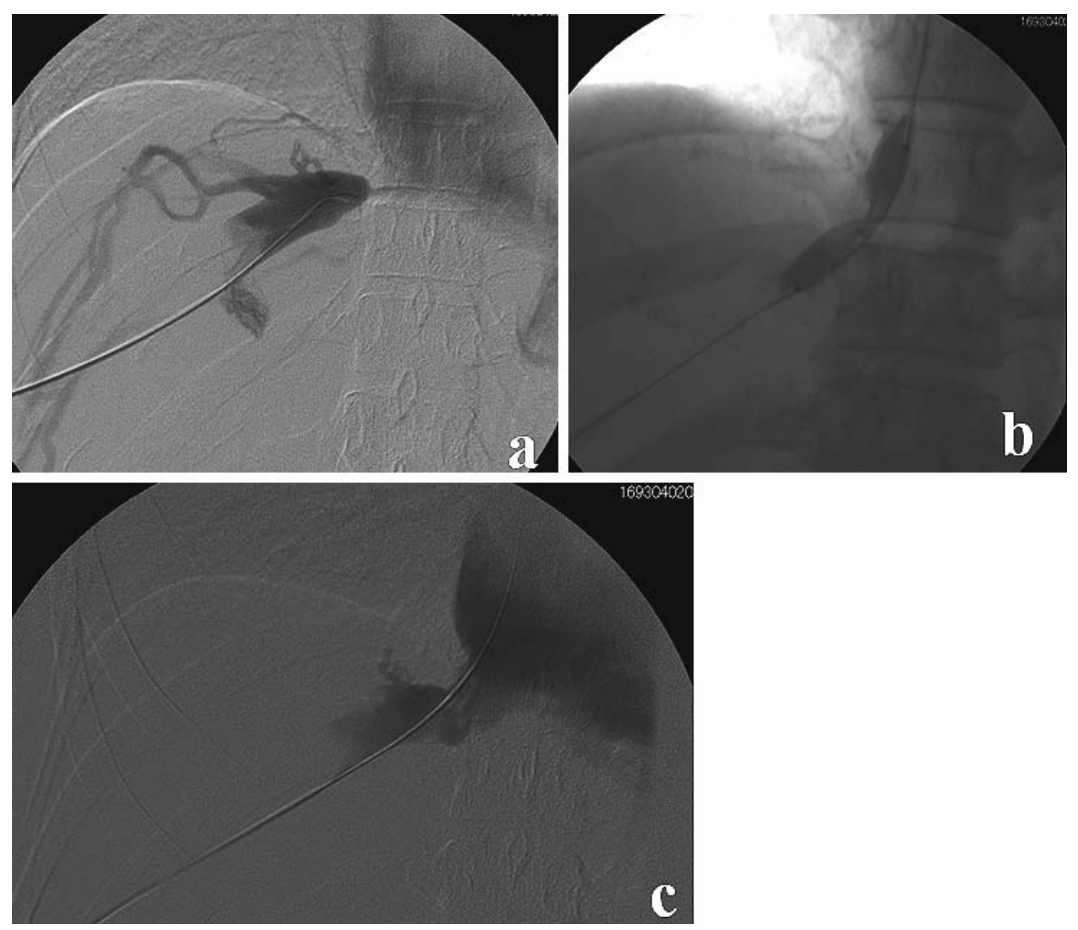

Figure 5. X-ray venography of the right hepatic vein. Before treatment, the right hepatic vein showed marked narrowing; the contrast medium could not flow to the IVC from the right hepatic vein (a). A balloon catheter was dilated at the level of the hepatic vein stenosis (b). Just after angioplasty, the stenosis appeared to have improved (c).

agnosis of Budd-Chiari syndrome $(12,17,18)$. The flat pattern indicates that there is no central venous pressure effect. In the present case, the completely flat waveform changed to a waveform with a decreased amplitude and phasic oscillations after the first angioplasty in 2001. It is likely that the angioplasty was not very effective, since the waveform did not improve completely. Two papers reported that balloon angioplasty resulted in a change from a flat pattern to a phasic pattern in the hepatic vein $(16,19)$. With effective treatment, the Doppler waveform shows a triphasic pattern. After our patient's second angioplasty, the flat waveform changed to a triphasic pattern. Thus, the second angioplasty appeared to have been effective. In this context, Doppler sonography was useful for determining the effectiveness of treatment and for diagnosing re-stenosis.
Moreover, re-stenosis was diagnosed when the patient developed a flat wave pattern. Doppler sonography was useful for screening and evaluation of re-stenosis. By diagnosing and treating re-stenosis early, the patient's prognosis may be improved. While venography is the most reliable method for evaluating the effect of angioplasty or diagnosing re-stenosis (20), it is invasive, exposes the patient to radiation, and is expensive. However, Doppler sonography is an easy and non-invasive method that is useful for evaluating hepatic vein blood flow.

We report a case of Budd-Chiari syndrome in which Doppler sonography was useful for diagnosing hepatic vein restenosis and for evaluating the effectiveness of treatment. Patients with Budd-Chiari syndrome should be followed with Doppler sonography.

\section{References}

1. Fisher NC, McCafferty I, Dolapci M, et al. Managing Budd-Chiari syndrome: a retrospective review of percutaneous hepatic vein angioplasty and surgical shunting. Gut 44: 568-574, 1999.

2. Langlet P, Escolano S, Valla D, et al. Clinicopathological forms and prognostic index in Budd-Chiari syndrome. J Hepatol 39: 496-501, 2003.

3. Janssen HL, Garcia-Pagan JC, Elias E, et al. Budd-Chiari syndrome: a review by an expert panel. J Hepatol 38: 364-371, 2003.

4. Valla DC. The diagnosis and management of the Budd-Chiari syndrome: consensus and controversies. Hepatology 38: 793-803, 2003.

5. Dilawari JB, Bambery P, Chawla Y, et al. Hepatic outflow obstruction (Budd-Chiari syndrome). Experience with 177 patients and a review of the literature. Medicine 73: 21-36, 1994.
6. Ludwig J, Hashimoto E, McGill DB, van Heerden JA. Classification of hepatic venous outflow obstruction: ambiguous terminology of the Budd-Chiari syndrome. Mayo Clin Proc 65: 51-55, 1990.

7. Bogin V, Marcos A, Shaw-Stiffel T. Budd-Chiari syndrome: in evolution. Eur J Gastroenterol Hepatol 17: 33-35, 2005.

8. Lim JH, Park JH, Auh YH. Membranous obstruction of the inferior vena cava: comparison of findings at sonography, CT, and venography. AJR Am J Roentgenol 159: 515-520, 1992.

9. Inoue A, Michitaka K, Shigematsu S, et al. Budd-Chiari syndrome associated with hypereosinophilic syndrome; A case report. Intern Med 46: 1095-1100, 2007.

10. Kage M, Arakawa M, Kojiro M, Okuda K. Histopathology of membranous obstruction of the inferior vena cava in the Budd- 
Chiari syndrome. Gastroenterology 102: 2081-2090, 1992.

11. Chan P, Lee CP, Yang CY, Hung JS. Complete membranous obstruction of the inferior vena cava: clinical characteristics of Chinese patients. J Intern Med 234: 501-505, 1993.

12. Bolondi L, Gaiani S, Li Bassi S, et al. Diagnosis of Budd-Chiari syndrome by pulsed Doppler ultrasound. Gastroenterology 100: 1324-1331, 1991.

13. Rossi S, Rosa L, Ravetta V, et al. Contrast-enhanced versus conventional and color Doppler sonography for the detection of thrombosis of the portal and hepatic venous systems. AJR Am J Roentgenol 186: 763-773, 2006.

14. Tavill AS, Wood EJ, Kreel L, Jones EA, Gregory M, Sherlock S. The Budd-Chiari syndrome: correlation between hepatic scintigraphy and the clinical, radiological, and pathological findings in nineteen cases of hepatic venous outflow obstruction. Gastroenterology 68: 509-518, 1975.

15. Bolondi L, Li Bassi S, Gaiani S, et al. Liver cirrhosis: changes of Doppler waveform of hepatic veins. Radiology 178: 513-516, 1991.
16. Ohta M, Hashizume M, Tomikawa M, Ueno K, Tanoue K, Sugimachi K. Analysis of hepatic vein waveform by Doppler ultrasonography in 100 patients with portal hypertension. Am J Gastroenterol 89: 170-175, 1994.

17. Hosoki T, Kuroda C, Tokunaga K, Marukawa T, Masuike M, Kozuka T. Hepatic venous outflow obstruction: evaluation with pulsed duplex sonography. Radiology 170 (3 Pt 1): 733-737, 1989.

18. Ralls PW, Johnson MB, Radin DR, Boswell WD Jr, Lee KP, Halls JM. Budd-Chiari syndrome: detection with color Doppler sonography. AJR Am J Roentgenol 159: 113-116, 1992.

19. Murashima N, Takeuchi K, Nishi $Y$, et al. Color doppler imaging Budd-Chiari syndrome before and after percutaneous transluminal angioplasty: A case report. Diagnostic imaging of the abdomen 1990 10: 431-437, 1990 (in Japanese).

20. Brancatelli G, Vilgrain V, Federle MP, et al. Budd-Chiari syndrome: spectrum of imaging findings. AJR Am J Roentgenol 188: W168-W176, 2007.

(C) 2008 The Japanese Society of Internal Medicine http://www.naika.or.jp/imindex.html 